\title{
Trabajo en equipo y formación continua en estudiantes del doctorado en educación de la UNMSM
}

\author{
Teamwork and continuous training in students of the doctorate in education at the UNMSM
}

Trabalho em equipe e formação contínua em alunos do doutorado em educação da UNMSM

Luis Héctor Huamaní Huamán
lhuamanih@unmsm.edu.pe

Universidad Nacional Mayor de San Marcos, Lima-Perú

\author{
Ada Lucía Gallegos Ruíz Conejo \\ ada.gallegos@yahoo.com \\ https://orcid.org/0000-0002-8264-711X
}

Universidad Nacional Mayor de San Marcos, Lima-Perú

Artículo recibido 29 de octubre 2021, arbitrado y aceptado 20 de noviembre 2021 y publicado 30 de diciembre 2021

\author{
RESUMEN
}

ABSTRACT

RESUMO

El trabajo en equipo es concebido como una estrategia de aprendizaje que permite la actualización continua de los diferentes contenidos procedimentales, actitudinales y conceptuales de los docentes. En este sentido, el objetivo planteado fue establecer la relación existente entre el trabajo en equipo y la formación continua en los estudiantes del Doctorado en Educación y Docencia Universitaria de la Universidad Nacional Mayor de San Marcos. La metodología del presente trabajo tiene un enfoque cuantitativo, el alcance de la investigación es descriptiva correlacional y cuenta con un diseño no experimental de tipo transversal. El resultado del Chi cuadrado de Pearson del cuestionario como de la escala Likert es menor a 0.05. Esto permite concluir que la variable independiente $\mathrm{y}$ dependiente guardan relación positiva. Además, el coeficiente de correlación de Spearman revela un mayor grado de correlación entre las variables.

Palabras clave: Trabajo en equipo; Formación continua; Habilidad; Capacidad; Competencias
Teamwork is conceived as a learning strategy that allows the continuous updating of the different procedural, attitudinal and conceptual contents of teachers. In this sense, the objective was to establish the existing relationship between teamwork and continuous training in students of the Doctorate in Education and University Teaching at the Universidad Nacional Mayor de San Marcos. The methodology of the present work has a quantitative approach, the scope of the research is correlational descriptive and has a non-experimental cross-sectional design. The result in Pearson's Chi square in his questionnaire and in his Likert scale is less than 0.05 . This allows us to conclude that the independent and dependent variables are positively related. Furthermore, the Spearman correlation coefficient reveals a higher degree of correlation between the variables.

Key words: Teamwork; Continuous training; Skills; Capacity; Competence
O trabalho em equipe é concebido como uma estratégia de aprendizagem que permite a atualização contínua dos diferentes conteúdos procedimentais, atitudinais e conceituais dos professores. Neste sentido, o objetivo era estabelecer a relação entre o trabalho em equipe e a formação contínua dos alunos do Doutorado em Educação e Ensino Universitário da Universidade Nacional Mayor de San Marcos. A metodologia deste trabalho tem uma abordagem quantitativa, o escopo da pesquisa é descritivo correlacional e tem um desenho transversal não experimental. O resultado do Qui-quadrado de Pearson do questionário e da escala Likert é inferior a 0,05. Isto nos permite concluir que as variáveis independentes e dependentes estão positivamente relacionadas. Além disso, o coeficiente de correlação de Spearman revela um maior grau de correlação entre as variáveis.

Palavras-chave: Trabalho em equipe; Aprendizagem ao longo da vida; Habilidade; Habilidade; Competência; Competências 


\section{INTRODUCCIÓN}

El hombre desde su aparición y evolución en la historia de la humanidad tuvo que trabajar de manera mancomunada para poder dominar la naturaleza y de esta forma satisfacer sus necesidades. Esto implicó un mayor grado de manipulación sobre su medio y un mayor conocimiento de esta para poder subsistir a las adversidades que se le presentaba conforme iba evolucionando en su desplazamiento del África hacia otros continentes. En todo este proceso histórico el ser humano no lidió solo contra la realidad circundante, sino lo hizo en coordinación con otros miembros de su misma especie lo cual le permitió obtener resultados eficientes, es decir, mejoró sus diferentes habilidades y técnicas para dominar y transformar su medio.

En las últimas décadas se observa que diferentes investigadores e instituciones han abordado la formación docente como un indicador indispensable de la calidad de los servicios educativos que ofrecen las instituciones. Se entiende que son múltiples las causas que afectan la formación continua del docente, pero el objetivo de la tesis es establecer la relación existente entre el trabajo en equipo y la formación continua de los estudiantes del Doctorado en Educación y Docencia Universitaria de la Universidad Nacional Mayor de San Marcos. Así mismo, el presente estudio tiene como justificación ampliar la perspectiva teórica y práctica del trabajo en equipo como un paradigma que permite la actualización de los docentes en una sociedad tan dinámica.

El trabajo en equipo no es nuevo para la especie humana; sino una forma de actuar estratégicamente para enfrentar los retos y alcanzar los objetivos organizacionales de forma eficaz y eficiente. En este sentido, Acosta (2014) define a un equipo como "grupo de personas que aúnan sus esfuerzos para alcanzar un objetivo común" (p.75). Esto implica una disposición a trabajar y aprender juntos, esto es, a desarrollar habilidades mientras se coordina o planifica las actividades pedagógicas. En el enfoque de Katzenbach y Smith citado por Ronco (2018) precisan que un equipo es "un número pequeño de personas con habilidades complementarias y comprometidas con un propósito común, por las que se consideran mutuamente responsables de su cumplimiento" (p. 20).

La interacción formal entre los docentes en los centros educativos son producto de una planificación institucional que busca generar un contexto social óptimo para el aprendizaje continuo de sus integrantes como consecuencia de los conflictos cognitivos, preguntas y repreguntas que surge en el proceso del trabajo mancomunado. Los conflictos funcionales deben gestionarse y promoverse desde la alta dirección con la finalidad de innovar y lograr los objetivos de la práctica pedagógica. Esto es, producir ventajas competitivas que permiten a la institución educativa fortalecer o crear una cultura organizacional que se fundamente en la interacción de personas, trabajo en equipo, para poder obtener alternativas de solución frente a los requerimientos educativos y de la comunidad.

El profesorado se congrega de forma intencional cuando hay objetivos organizacionales que cumplir $y$ en el proceso de lograr la meta se expresan diferentes problemas que tienen que ser gestionados con precisión y anticipación. Arbaiza (2017, p.245) propone que "para que un equipo de trabajo sea exitoso en términos de productividad, desempeño, innovación y satisfacción de sus miembros, debe desarrollarse en un contexto organizacional que provea los recursos físicos, económicos y 
tecnológicos necesario para la realización de tareas". Para el logro de las metas es necesario contar con un capital tangible como se mencionó, pero también se requiere un capital intangible como la comunicación efectiva, el liderazgo, la gestión de conflictos y sobre todo aprender a trabajar en equipo.

Los equipos de trabajo no surgen de forma espontánea debido a que su formación requiere de un conjunto de condiciones que tienen que lograr sus integrantes para hacerse y considerarse como tal. Además, una vez logrado el objetivo se tiene que seguir manteniendo las condiciones de sincronización para no dejar de ser un equipo. En un enfoque organizacional se afirmar que su origen se encuentra en la formación de grupos de trabajo, en este sentido Robbins \& Coulter (2018, p.418) formulan que "un grupo se define como dos o más individuos que interactúan y son interdependientes, que se reúne para lograr, metas específicas”. Esta concepción confirma que los grupos que logren gestionar eficientemente sus emociones, sus conflictos, su liderazgo, su cultura organizacional, su compromiso institucional y su mejora comunicativa, entonces, habrán pasado a ser un equipo de trabajo.

Lograr la formación de equipos de trabajo no implica renunciar al trabajo individual, por el contrario, se requiere de este aporte. El trabajo individual enriquece el trabajo mancomunado porque se convierte en insumo, por lo tanto, son complementarios. Hay que tener en cuenta que la esencia del trabajo en equipo se sostiene en el aporte que puede brindar cada uno de sus miembros, es decir, el conocimiento y la investigación continua de sus integrantes aseguran un aprendizaje continuo del resto. En este sentido, el aprendizaje colaborativo y cooperativo juega un papel fundamental en la construcción del conocimiento y en el desarrollo de habilidades básicas y específicas. No obstante, desde una perspectiva tradicional se les consideran como dos categorías opuestas.

La comunicación es un proceso que se realiza diariamente para satisfacer las necesidades humanas y al mismo tiempo es tan compleja que en muchos casos de análisis organizacionales solo se toma en cuenta la transferencia de información, esto es, se asume que la comunicación se realiza por el hecho de transferir información. En esto reside las causas de las frustraciones y fracasos de las instituciones que pudieron gestionar eficientemente su comunicación administrativa y pedagógica. Asimismo, cuando la comunicación llega al receptor, pero es obtusa en su comprensión. Entonces, se asevera que se aseguró la transferencia de información más no la comprensión.

La estructura de la información es una variable a considerar en los diferentes procesos de planeación, organización, dirección y control; debido a esta se asegura el traslado de información que permitirá el cumplimento de los propósitos de cada proceso. En este sentido, es la clave para el éxito pedagógico y la gestión de la institución educativa. Además, genera las condiciones para que el trabajo en equipo se convierta en un arte de crear momentos que permite la integración de sus miembros. Esto implica que la comunicación debe tener un propósito y debe ser planificada, esto es, debe ser estratégica para alcanzar la meta programada. La Pedagogía de la comunicación debe ser promovida con conciencia teórica para que la práctica tenga un fundamento científico.

Los docentes son consumidores y productores de información, por ello en los diferentes contextos pedagógicos el profesorado debe ser un buen comunicador y para ello debe ser prolijo en lo que dice y como lo dice, porque la comunicación es 
verbal y no verbal. La función de la comunicación permite entender que esta no solo es trasmisión de información, sino que genera cambios conductuales en las personas que lo recepciona. Al mismo tiempo energiza la conducta para la ejecución de propósitos, así como, permite expresar la vivencia, lo cual conlleva a establecer lazos más sólidos entre los integrantes. Esto le da un sentido humanista a las actividades que realizan y por último permite la toma de decisiones consensuadas de los miembros del equipo de trabajo.

Robbins y Coulter (2018) sostienen que "un líder es la persona que puede influenciar en los demás y que tiene autoridad administrativa. El liderazgo es el proceso de dirigir a un grupo de influir en él para que alcance sus metas. Esto lo hacen los líderes" (p.555). Los equipos de trabajo tienen como finalidad plantear resultados o alternativas a la institución educativa en base a la demanda o necesidades de la sociedad, por esta razón, se debe promover un liderazgo para el cambio continuo. En este contexto las instituciones educativas requieren reinventarse en todos sus procesos de organización y ejecución para conseguir lo propósitos trazados, esto es, tener vigencia en la comunidad y asegurar la sostenibilidad de la calidad del servicio educativo que brinda.

La formación continua del profesorado es catalogada con diversos nombres como formación permanente o formación para la toda la vida. Para entender su conceptualización y su práctica en el quehacer pedagógico, Imbernón (2017) lo define como "acciones formativas, en distintas modalidades, que el profesorado va cursando a lo largo de su vida a través del contacto con los compañeros de trabajo, el ambiente de clase y del centro educativo y la interacción con el alumnado" (p.71). A partir de esta conceptualización se ratifica que el docente se consolida académicamente a través del tiempo, es decir, aprende de diferentes contextos y circunstancias ya sea formales $\mathrm{e}$ informales. Sin embargo, este proceso debe estar acompañado de la reflexión pedagógica para poder consolidar la adquisición de saberes, de lo contrario esos conocimientos no serán aprovechados.

La sociedad demanda por docentes altamente competitivos para el desarrollo integral de las futuras generaciones que aportarán sus saberes y habilidades a generar bienestar en sus comunidades. Por ello, se torna una necesidad en la aldea global de contar con profesionales que dominen contenidos de su asignatura y hayan desarrollado competencias genéricas y específicas. Esto permitirá que el docente tenga las condiciones o herramientas que le ayudará a enseñar y desarrollar competencias en sus alumnos. En este sentido, Trahtemberg (2014) expresa que "el techo del maestro marca el techo del alumno" (p. 136) de su afirmación podemos inferir que una de las aristas que cobra una vital importancia en la mejora del sistema educativo es la calidad del docente.

Los docentes que buscan estar en la vanguardia académica de su profesión regulan su formación gestionando su tiempo, aprendizaje y su reflexión, esto es, dedicándose a cultivar su saber pedagógico, así como planificando sus sesiones de aprendizaje. Por ello, Imbernón et al. (2016) plantea que "algunas de las características definitorias de la formación son su intencionalidad pedagógica y su potencialidad para diseñar y desarrollar dispositivos que permitan la adquisición de aprendizajes relevantes. Es decir, una actividad dirigida a formar a otros para mejorar" (p. 13). El hecho de preparar una clase y compartirla con los educandos genera 
un contexto propicio donde la reflexión pedagógica en interacción con las preguntas o respuestas de los alumnos conlleva a la mejora continua.

La formación académica del profesorado es permanente, ha sido así en la historia de la humanidad y lo seguirá siendo en la Historia del desarrollo cultural del hombre porque especie humana tiene por característica transformar su medio y este le exige su transformación. Por este motivo, Souto (2016) expresa que un "profesional de la enseñanza no termina de formarse, aunque concluya ciclos de formación inicial o continua; el proceso continúa en otros espacios de formación, de desempeño profesional y de profesionalización in situ, a la vez que se extiende a otros" (p. 67). De lo expresado, se infiere que las políticas de formación docente deben de estar enfocado capacitaciones o talleres, así como, en la creación de una cultura organizacional que permitan la mejora continua del talento docente.

La formación continua del profesorado debe acompañarse de la reflexión pedagógica desde su preparación universitaria y esta se debe caracterizar por ser sistemática. Imbernón et al. (2016) propone que "la formación como disciplina ha de reflexionar sobre sí misma y sobre sus posibilidades de transmisión" (p. 13). Esto reafirmar que la formación del profesorado necesita la formalización de cursos que teoricen y pongan en práctica su mejora continua. Esto coadyuvaría a sensibilizar esta práctica tan antigua, filosofar sobre sus acciones educativas, pero poca practicada por una inadecuada gestión del tiempo y por la acción rutinaria en la que han caído muchos docentes la cual representa la renuncia a seguir aportando a su profesión como a su aprendizaje.

\section{MÉTODO}

La investigación se ha desarrollado con un enfoque cuantitativo la cual tiene un alcance descriptivo correlacional, mientras que la estrategia empleada para responder las preguntas de investigación, así como probar la hipótesis ha sido el diseño no experimental. Para el estudio de la variable independiente, trabajo en equipo, y dependiente, formación continua, se aplicaron dos instrumentos, cuestionario y escala Likert, con la finalidad de lograr la validez de criterio. Para obtener la validez de contenido y de constructo de los instrumentos se procedió a validarlos mediante juicios de expertos. La confiabilidad se estableció mediante el alfa de Cronbach. Además, la población de estudio estuvo constituida por los estudiantes del doctorado en educación de la Universidad Nacional Mayor de San Marcos la cual estaba conformada por dos secciones que sumaban 30 estudiantes. Por el diseño de investigación se procedió aplicar los instrumentos de investigación en los grupos ya formados cuyos miembros poseen experiencia en el trabajo de aula como parte de sus actividades pedagógicas diarias.

\section{RESULTADOS}

\section{Objetivos específicos-01}

OE1. Comprobar la relación existente entre el trabajo en equipo y la formación continua de los estudiantes del Doctorado en Educación y Docencia Universitaria de la Universidad Nacional Mayor de San Marcos. 
Tabla 1. X: Trabajo en Equipo (Cuestionario) ${ }^{\star}$ Y: Formación Continua (Cuestionario) tabulación cruzada.

\begin{tabular}{|c|c|c|c|c|c|}
\hline & & & \multicolumn{2}{|c|}{ Y: Formación Continua (Cuestionario) } & \multirow[b]{2}{*}{ Total } \\
\hline & & & Están de acuerdo & $\begin{array}{l}\text { No están de } \\
\text { acuerdo }\end{array}$ & \\
\hline \multirow{4}{*}{$\begin{array}{c}\text { X: Trabajo } \\
\text { en Equipo } \\
\text { (Cuestionario) }\end{array}$} & \multirow[b]{4}{*}{$\begin{array}{l}\text { No están de } \\
\text { acuerdo }\end{array}$} & Recuento & 11 & 2 & 13 \\
\hline & & $\begin{array}{l}\text { \% dentro de X: Trabajo } \\
\text { en Equipo (Cuestionario) }\end{array}$ & $84.6 \%$ & $15.4 \%$ & $100.0 \%$ \\
\hline & & Recuento & 3 & 14 & 17 \\
\hline & & $\begin{array}{l}\text { \% dentro de X: Trabajo } \\
\text { en Equipo (Cuestionario) }\end{array}$ & $17.6 \%$ & $82.4 \%$ & $100.0 \%$ \\
\hline \multirow{2}{*}{\multicolumn{2}{|c|}{ Total }} & Recuento & 14 & 16 & 30 \\
\hline & & $\begin{array}{l}\text { \% dentro de X: Trabajo } \\
\text { en Equipo (Cuestionario) }\end{array}$ & $46.7 \%$ & $53.3 \%$ & $100.0 \%$ \\
\hline
\end{tabular}

La Tabla 1 muestra una tabla de doble entrada de las variables que participaron en los diferentes niveles. Por ejemplo 11 personas están de acuerdo y 14 personas no están de acuerdo, refiriéndose a la concordancia de respuestas en los instrumentos de trabajo en equipo y la formación continua.

\section{Hipótesis específicas-01}

HE1. El trabajo en equipo colaborativo se relaciona con la formación continua de los estudiantes del Doctorado en Educación y Docencia Universitaria de la Universidad Nacional Mayor de San Marcos

Tabla 2. Pruebas de chi-cuadrado para el contraste de dependencia.

\begin{tabular}{|c|c|c|c|c|c|}
\hline & Valor & gl & $\begin{array}{l}\text { Sig. asintótica } \\
\text { ( } 2 \text { caras })\end{array}$ & $\begin{array}{c}\text { Significación } \\
\text { exacta ( } 2 \text { caras })\end{array}$ & $\begin{array}{l}\text { Significación } \\
\text { exacta ( } 1 \text { cara) }\end{array}$ \\
\hline Chi-cuadrado de Pearson & $13,274 a$ & 1 & 000 & & \\
\hline Corrección de continuidadb & 10,720 & 1 & 001 & & \\
\hline Razón de verosimilitud & 14,449 & 1 &, 000 & & \\
\hline Prueba exacta de Fisher & & & & 001 & ,000 \\
\hline Asociación lineal por lineal & 12,832 & 1 &, 000 & & \\
\hline $\mathrm{N}$ de casos válidos & 30 & & & & \\
\hline
\end{tabular}

\section{Hipótesis estadístico}

H0: No Existe relación entre el trabajo en equipo y la formación continua de los estudiantes del Doctorado en Educación y Docencia Universitaria
H1: Existe relación entre el trabajo en equipo y la formación continua de los estudiantes del Doctorado en Educación y Docencia Universitaria 


\section{Nivel de significancia}

Con $\alpha=0.05$ nivel de significancia

Decisión Si p-valor $=0.00<a=0.05$ rechaza $\mathrm{H} 0$ y se acepta $\mathrm{H} 1$ : Existe relación entre el trabajo en equipo y la formación continua de los estudiantes del Doctorado en Educación y Docencia

Tabla 3. Coeficiente de contingencia (grado de relación).

\begin{tabular}{lcccc}
\hline & Valor & $\begin{array}{c}\text { Error } \\
\text { estándar } \\
\text { asintótica }\end{array}$ & Aprox. S & $\begin{array}{c}\text { Aprox. } \\
\text { Sig. }\end{array}$ \\
\hline Nominal por Nominal coeficiente de contingencia &, 554 & &, 000 \\
Intervalo por intervalo R de persona &, 665 &, 136 & 4,714 & 000 \\
Ordinal por Ordinal correlación de Spearman &, 665 &, 136 & 4,714 & 000 \\
N de casos válidos & 30 & & & \\
\hline
\end{tabular}

En la Tabla 3 se observa el coeficiente de contingencia de $55.4 \%$ de dependencia o relación entre el trabajo en equipo y la formación continua de los estudiantes del Doctorado en Educación y Docencia Universitaria. Es decir, la mayoría de los estudiantes estuvieron de acuerdo con el trabajo en equipo y la formación continua. Considerando el orden de respuesta la correlación de Spearman es $66.5 \%$ de relación entre las variables de estudio. Utilizando el instrumento del cuestionario.

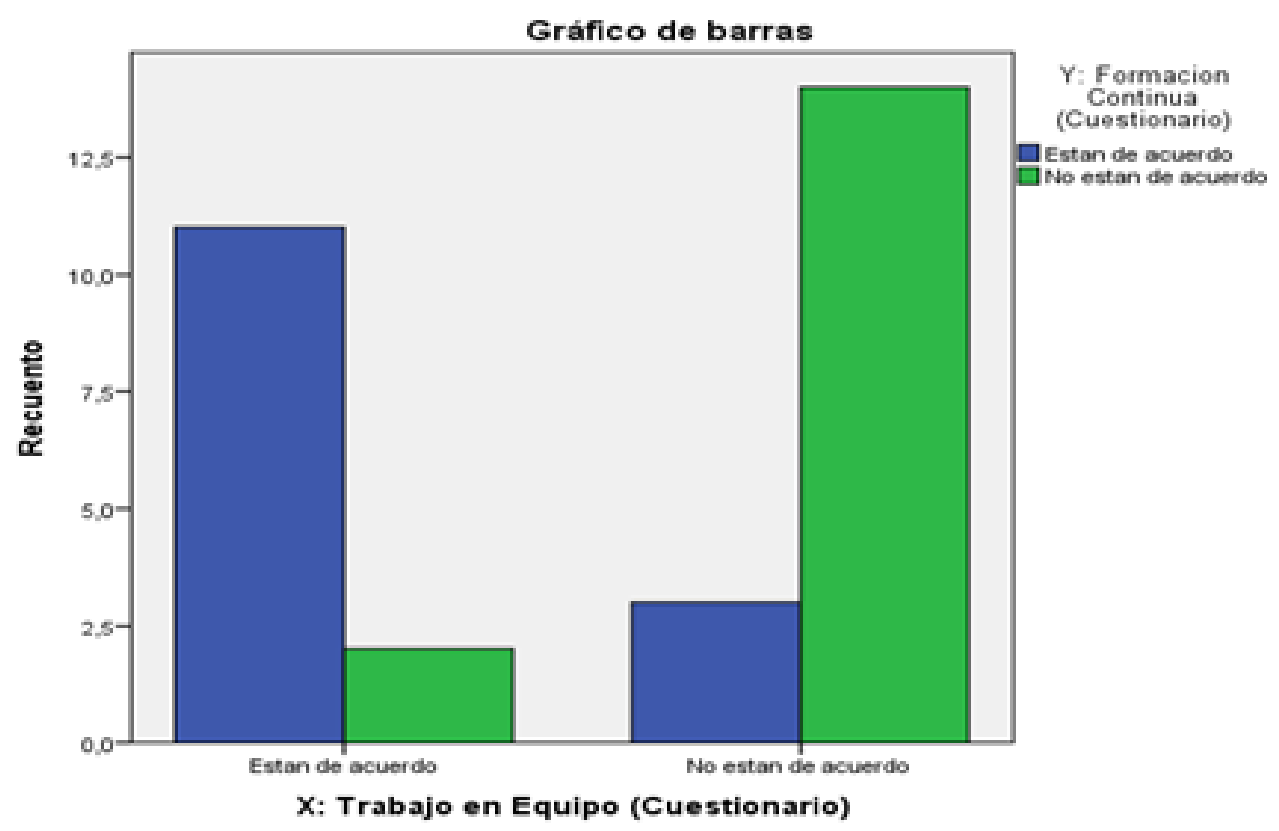

Gráfico 1. Trabajo en Equipo. 
El Gráfico 1 muestra las respuestas de acuerdo a las preguntas de si estaban de acuerdo o no estaban de acuerdo. De acuerdo a las variables de trabajo en equipo y la formación continua de los estudiantes del Doctorado en Educación y Docencia Universitaria.

\section{Objetivos específicos-02}

OE2 Describir la relación existente entre el trabajo en equipo y la formación continua de los estudiantes del Doctorado en Educación y Docencia Universitaria de la Universidad Nacional Mayor de San Marcos en Perú.

Tabla 4. Trabajo en equipo y formación continua (tabulación).

\begin{tabular}{|c|c|c|c|c|c|c|c|}
\hline & & & Y: Forn & n Conti & (Escala de & kert) & \\
\hline & & & $\begin{array}{c}\text { En } \\
\text { desacuerdo }\end{array}$ & Neutro & De acuerdo & $\begin{array}{l}\text { Totalmente } \\
\text { de acuerdo }\end{array}$ & Total \\
\hline \multirow{10}{*}{$\begin{array}{l}\text { X: Trabajo } \\
\text { en Equipo } \\
\text { (Escala de } \\
\text { Likert) }\end{array}$} & \multirow[b]{2}{*}{$\begin{array}{l}\text { Totalmente } \\
\text { desacuerdo }\end{array}$} & Recuento & 1 & 0 & 0 & 0 & 1 \\
\hline & & $\begin{array}{l}\text { \% dentro de X: } \\
\text { Trabajo en Equipo } \\
\text { (Escala de Likert) }\end{array}$ & $100.0 \%$ & $0.0 \%$ & $0.0 \%$ & $0.0 \%$ & $100.0 \%$ \\
\hline & \multirow[b]{2}{*}{$\begin{array}{c}\text { En } \\
\text { desacuerdo }\end{array}$} & Recuento & 3 & 1 & 0 & 0 & 4 \\
\hline & & $\begin{array}{l}\text { \% dentro de X: } \\
\text { Trabajo en Equipo } \\
\text { (Escala de Likert) }\end{array}$ & $75.0 \%$ & $25.0 \%$ & $0.0 \%$ & $0.0 \%$ & $100.0 \%$ \\
\hline & \multirow[b]{2}{*}{ Neutro } & Recuento & 0 & 7 & 0 & 0 & 7 \\
\hline & & $\begin{array}{l}\text { \% dentro de X: } \\
\text { Trabajo en Equipo } \\
\text { (Escala de Likert) }\end{array}$ & $0.0 \%$ & $100.0 \%$ & $0.0 \%$ & $0.0 \%$ & $100.0 \%$ \\
\hline & \multirow[b]{2}{*}{ De acuerdo } & Recuento & 0 & 1 & 7 & 1 & 0 \\
\hline & & $\begin{array}{l}\text { \% dentro de X: } \\
\text { Trabajo en Equipo } \\
\text { (Escala de Likert) }\end{array}$ & $0.0 \%$ & $11.1 \%$ & $77.8 \%$ & $11.1 \%$ & $100.0 \%$ \\
\hline & \multirow[b]{2}{*}{$\begin{array}{l}\text { Totalmente } \\
\text { de acuerdo }\end{array}$} & Recuento & 0 & 0 & 3 & 6 & 9 \\
\hline & & $\begin{array}{l}\text { \% dentro de X: } \\
\text { Trabajo en Equipo } \\
\text { (Escala de Likert) }\end{array}$ & $0.0 \%$ & $0.0 \%$ & $33.3 \%$ & $66.7 \%$ & $100.0 \%$ \\
\hline \multirow[b]{2}{*}{ Total } & & Recuento & 4 & 9 & 10 & 7 & 30 \\
\hline & & $\begin{array}{l}\text { \% dentro de X: } \\
\text { Trabajo en Equipo } \\
\text { (Escala de Likert) }\end{array}$ & $13.3 \%$ & $30.0 \%$ & $33.3 \%$ & $23.3 \%$ & $100.0 \%$ \\
\hline
\end{tabular}

La Tabla 4 destaca la doble entrada de las variables que participaron en los diferentes niveles considerando el instrumento de Likert. Por ejemplo, en la fila del total $(10+7=17)$ personas están de acuerdo y totalmente de acuerdo. Mientras 7 personas se mantienen neutro y en desacuerdo y totalmente en desacuerdo 6 personas. Es decir, la mayoría está de acuerdo con el uso del instrumento de Likert.

\section{Hipótesis específicas-02}

HE2 El trabajo en equipo cooperativo se relaciona con la formación continua de los estudiantes del Doctorado en Educación y Docencia Universitaria de la Universidad Nacional Mayor de San Marcos. 
Tabla 5. Pruebas de chi-cuadrado para el contraste de dependencia.

\begin{tabular}{lccc}
\hline & Valor & gl & Sig. asintótica (2 caras) \\
\hline Chi-cuadrado de Pearson & 55,864 & 12 &, 000 \\
Razón de verosimilitud & 51,874 & 12 &, 000 \\
Asociación lineal por lineal & 23,772 & 1 &, 000 \\
N de casos válidos & 30 & & \\
\hline
\end{tabular}

\section{Hipótesis estadístico}

H0: No Existe relación entre el trabajo en equipo y la formación continua de los estudiantes del Doctorado en Educación y Docencia Universitaria

H1: Existe relación entre el trabajo en equipo y la formación continua de los estudiantes del Doctorado en Educación y Docencia Universitaria.

\section{Nivel de significancia}

Con $\alpha=0.05$ nivel de significancia

Decisión: Si p-valor $=0.00<\alpha=0.05$

Se rechaza $\mathrm{H} 0$ y se acepta H1: Existe relación entre el trabajo en equipo y la formación continua de los estudiantes del Doctorado en Educación y Docencia Universitaria.

Tabla 6. Coeficiente de contingencia (grado de relación).

\begin{tabular}{|c|c|c|c|c|c|}
\hline & & Valor & $\begin{array}{c}\text { Error } \\
\text { estándar } \\
\text { asintótico }\end{array}$ & Aprox. S & Aprox. Sig. \\
\hline Nominal por Nominal & Coeficiente de contingencia & ,807 & & &, 000 \\
\hline Intervalo por intervalo & $\mathrm{R}$ de persona & ,905 & 033 & 11,283 &, $000 \mathrm{c}$ \\
\hline Ordinal por ordinal & Correlación de Spearman & ,906 & ,042 & 11,355 &, $000 c$ \\
\hline $\mathrm{N}$ de casos válidos & & 30 & & & \\
\hline
\end{tabular}

En la Tabla 6 se observa el coeficiente de contingencia de $80.7 \%$ de dependencia o relación entre el trabajo en equipo y la formación continua de los estudiantes del Doctorado en Educación y Docencia Universitaria. Es decir, la mayoría de los estudiantes están de acuerdo con el trabajo en equipo y la formación continua. Considerando el orden de respuesta la correlación de Spearman es 90.6\% de relación entre las variables de estudio. Utilizando el instrumento de medición de la escala de Likert. 


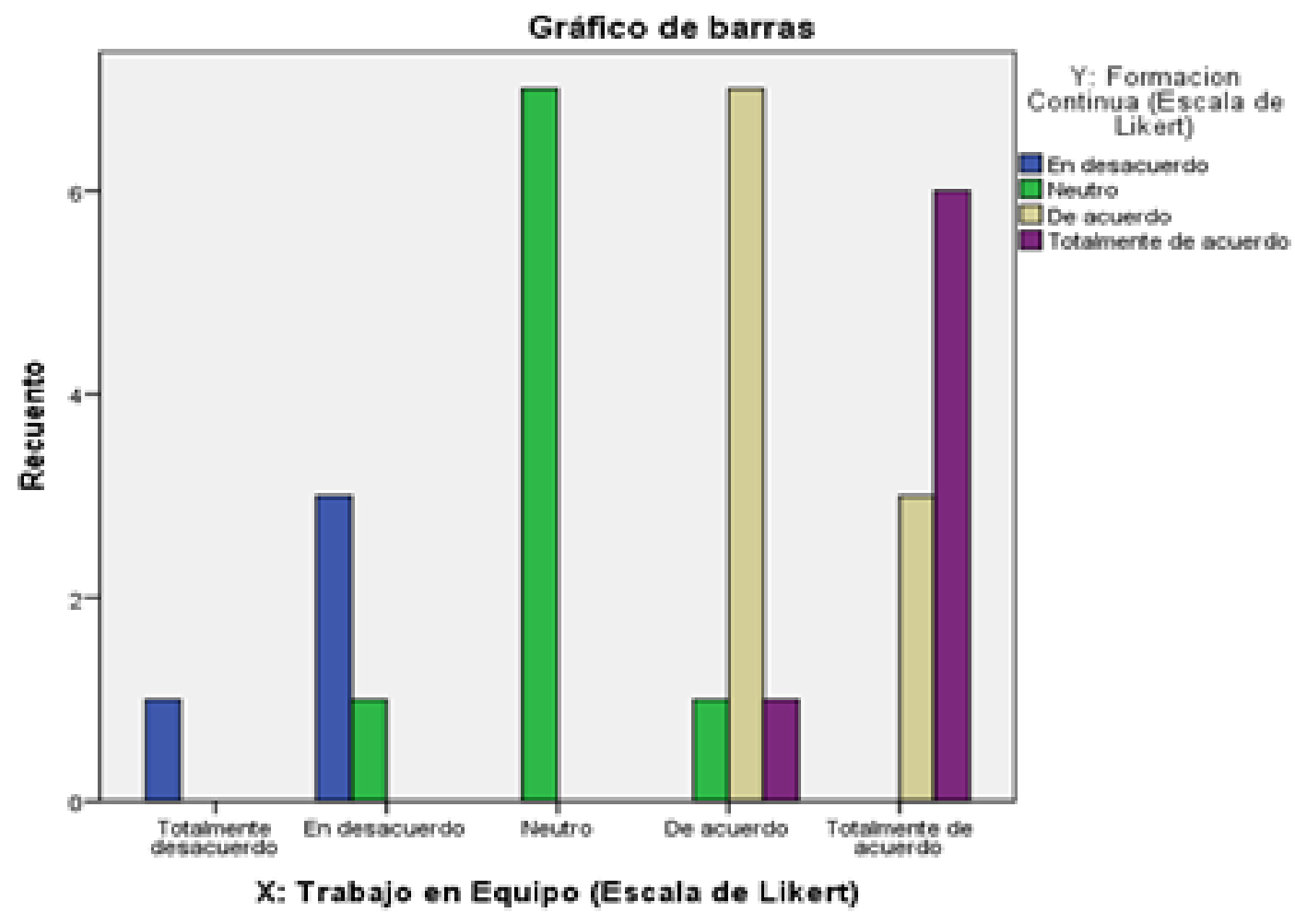

Gráfico 2. Trabajo en equipo en la escala de Likert.

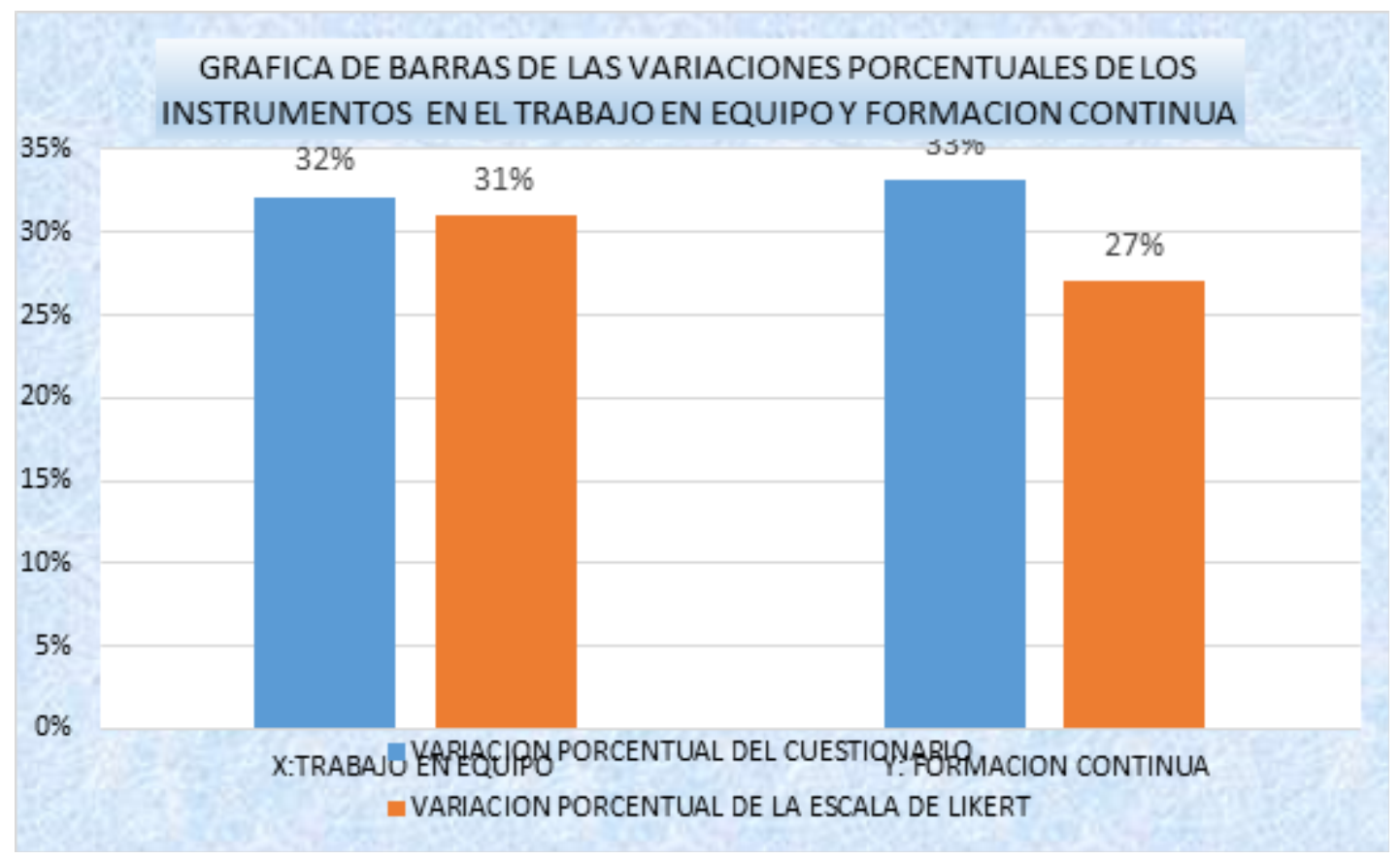

Gráfico 3. El trabajo en equipo y formación continua. 
Gráfico 3 muestra el porcentaje de variación del uso de los 2 instrumentos. Por ejemplo, dentro de la formación continua de acuerdo a los porcentajes todavía no están acostumbrados con el instrumento de Likert. Mientras en el uso del instrumento de cuestionario la mayoría conoce y está de acuerdo.

\section{Discusión}

Los hallazgos estadísticos encontrados, permiten rechazar la hipótesis nula y aceptar la hipótesis alterna, que establece una relación significativa entre el trabajo en equipo y la formación continua de los estudiantes del Doctorado en Educación y Docencia Universitaria de la Universidad Nacional Mayor de San Marcos. Por otro lado, el análisis estadístico no paramétrico aplicado a esta investigación da como resultado tanto en su cuestionario como en su escala Likert una relación significativa. El resultado del Chi cuadrado de Pearson en ambos instrumentos es menor a 0.05 , por lo cual se concluye que las variables, independiente y dependiente, guardan relación positiva. Además, el coeficiente de correlación de Spearman revela un mayor grado de correlación entre las variables.

Cárdenas (2016) en su tesis de maestría titulado "los docentes formados en la estrategia investigación- acción: percepciones sobre la mejora de su práctica pedagógica" lo que denota que la investigación-acción mejora la práctica pedagógica de los docentes entrevistados en la planificación, desarrollo de capacidades, trabajo en equipo, la aplicación de situaciones problemáticas contextualizadas, y el uso de recursos y materiales educativos en sus sesiones de aprendizaje. Asimismo, generó en ellos conocimientos nuevos, como las diversas estrategias de enseñanza para el desarrollo de sus sesiones de clase. Lo que indica que el uso de estrategias de manera positiva tal como lo demuestra este estudio contribuye en la mejora dentro del proceso de enseñanza y aprendizaje

Fernández (2015) en su tesis de doctorado titulado "Clima organizacional y calidad en el desempeño profesional de los docentes de la Facultad de Ciencias Administrativas y Turismo de la Universidad Nacional San Antonio de Abad" establece como problema: ¿cuál es la relación que existe entre el clima organizacional y la calidad del desempeño profesional de los docentes de la Facultad de Ciencias Administrativas y Turismo de la UNSAC? Y en base a los resultados comprobó cuantitativamente que la relación del clima organizacional y el desempeño docente de la Facultad tiene una correlación positiva $(\mathrm{r}=$ 0.9996). Por lo tanto, se debe tomar mayor énfasis en cuidar la calidad del clima organizacional para tener mayores y mejores resultados de la enseñanza y aprendizaje. Concediendo con los datos arrojados en este estudio sobre la correlación positiva y conlleva un análisis entre las variables dependiente e independientes.

Para Herrera (2016) en su tesis de doctorado denominado "Perfil profesional de los equipos directivos de los centros escolares públicos de la comuna de Talca" presenta como problema: ¿Cuáles son las características profesionales y las necesidades formativas de los equipos directivos de la red educativa municipal de la comuna de Talca, VII Región del Maule, año 2015? Y concluye en que las competencias de dirección y liderazgo son perceptibles (83\% del total de los encuestados), puesto que la dirección de los centros educativos 
se basa en la capacidad de delegar funciones, considerando los talentos y habilidades de sus miembros para entregar tareas. Estos directores manifiestan la intención de motivar para el logro de los aprendizajes y de los objetivos propuestos por el centro educativo.

Seguidamente Castro (2015) en su tesis de doctorado "La Formación Permanente del Profesorado Universitario: Análisis del Diseño y Desarrollo de los Procesos de Formación que Ofrece el Instituto de Profesionalización y Superación Docente de la Universidad Nacional Autónoma de Honduras" plantea como problema: ¿Cuáles son los criterios considerados para establecer la planificación, el diseño, la implementación y la evaluación de la formación permanente del profesorado universitario en la UNAH, a partir de la propuesta formativa del IPSD? Y concluye que los profesores manifiestan que el proceso de acompañamiento debe institucionalizarse para que logre impactar de la manera esperada; para ello, deben generarse equipos de trabajo y contar con el apoyo de las estructuras organizativas.

\section{CONCLUSIONES}

El trabajo en equipo incide significativamente en la formación continua de los docentes. Eso es posible porque la estrategia genera las condiciones para socializar contenidos y habilidades, es decir, permite el aprendizaje personalizado de sus integrantes. Asimismo, se observa que un equipo de trabajo se forma en un proceso donde sus integrantes sincronizan sus conocimiento, capacidades y valores. Esto tiene como etapa inicial la creación de un grupo de trabajo. Además, los equipos que logren constituirse siempre tendrán el riesgo de perder su condición, por ello la gestión de la sincronización se torna vital. También, los docentes conciben que la gestión de conflicto, el liderazgo, la comunicación organizacional permiten que los equipos logren sus objetivos de forma eficiente y eficaz. Sin embargo, se observan que los grupos pueden lograr objetivos con baja calidad.

\section{REFERENCIAS}

Acosta, J. M. (2014). Trabajo en equipo. Alfaomega

Arbaiza, F. L. (2017). Liderazgo y comportamiento organizacional. Editorial Universidad ESAN

Cárdenas Álvarez, A (2016). Los docentes formadores en la estrategia investigación acción: percepciones sobre la mejora sobre su práctica pedagógica. Tesis de maestría. PUCP. file:///C:/Users/HP/Downloads/CARDENAS_ ALVAREZ_CARMEN_LOS_DOCENTES_ FORMADOS.pdf

Castro González, O. (2015). La Formación Permanente del Profesorado Universitario: Análisis del Diseño y Desarrollo de los Procesos de Formación que Ofrece el Instituto de Profesionalización y Superación Docente de la Universidad Nacional Autónoma de Honduras. Tesis de doctorado. Universidad de Sevilla. https://idus.us.es/bitstream/ handle/11441/40042/3\%20Tesis\%20Olvis\%20 Castro.pdf? sequence $=1$ \&isAllowed $=y$

Delgado Santa Gadea, K. (2011). Aprendizaje colaborativo, teoría y práctica. Editorial San Marcos

Fernández Baca, M. G. (2015). Clima organizacional y calidad en el desempeño profesional de los docentes de la Facultad de Ciencias Administrativas y Turismo de la Universidad Nacional San Antonio de Abad. Tesis de doctorado. UNMSM. https://cybertesis.unmsm. edu.pe/handle/20.500.12672/9246? show=full

Herrera Martínez, F. (2016). Perfil profesional de los equipos directivos de los centros escolares públicos de la comuna de Talca. Tesis doctoral. Universidad dedAlcalá. https://ebuah.uah.es/dspace/bitstream/ 
handle/10017/29477/Tesis\%20Fernando $\% 20$ Alejandro\%20Herrera\%20Mart\%C3\%ADnez. pdf? sequence $=1$ \&isAllowed $=y$

Imbernón Muñoz, F., Medina Moya, J., Aránega Español, S., Jarauta Borrasca, B., y Serrat Antolí, N. (2016). Diseño, desarrollo y evaluación de los procesos de formación. Síntesis

Imbernón Muñoz, F. (2017). Ser docente en una sociedad compleja: la difícil tarea de enseñar.
GRAO

Robbins, S,. y Coulter, M. (2018). Administración. Pearson

Ronco, E. (2018). Equipos que crean valor en las organizaciones. PROFIT

Souto, M. (2016). Pliegues de la formación, sentido y herramientas para la formación.

Trahtemberg, L. (2014). Desaprender y reaprender. SM 